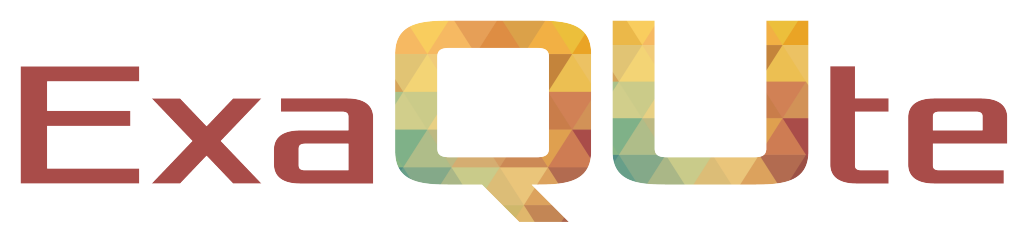

Exascale Quantification of Uncertainties for

Technology and Science Simulation

\title{
D4.3 Benchmarking report as tested on the available infrastructure
}

\section{Document information table}

\begin{tabular}{|l|l|}
\hline Contract number: & 800898 \\
\hline Project acronym: & ExaQUte \\
\hline Project Coordinator: & CIMNE \\
\hline Document Responsible Partner: & IT4I \\
\hline Deliverable Type: & Other \\
\hline Dissemination Level: & Public \\
\hline Related WP \& Task: & WP 4, Task 4.4 \\
\hline Status: & Final \\
\hline
\end{tabular}

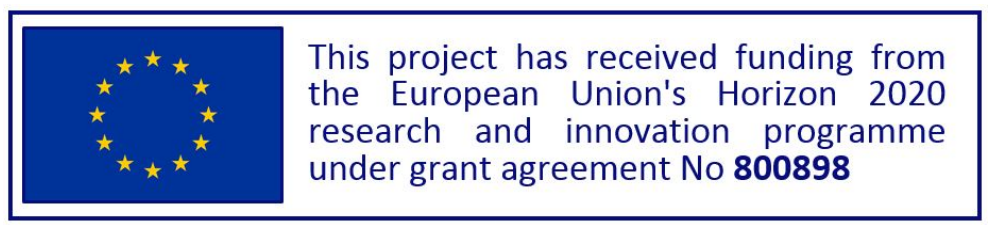




\section{Authoring}

\begin{tabular}{|l|l|l|l|l|}
\hline \multicolumn{5}{|l|}{ Prepared by: } \\
\hline Authors & Partner & Modified Page/Sections & Version & Comments \\
\hline Tomas Karasek & IT4I & & V0.1 & \\
\hline Stanislav Böhm & IT4I & & V0.1 & \\
\hline Brendan Keith & TUM & & V0.1 & \\
\hline Ramon Amela & BSC & & V0.2 & \\
\hline Rosa M Badia & BSC & & V0.2 & \\
\hline Tomas Karasek & IT4I & & V0.3 & \\
\hline Stanislav Böhm & IT4I & & V0.3 & \\
\hline \hline
\end{tabular}

\section{Change Log}

\begin{tabular}{|l|l|l|}
\hline Versions & Modified Page/Sections & Comments \\
\hline V0.1 & First document version & \\
\hline V0.2 & Results with PyCOMPSs and corrections & \\
\hline V0.2 & Results with HyperLoom and corrections & \\
\hline
\end{tabular}

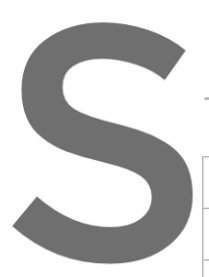

\section{Approval}

Aproved by:

Task leader
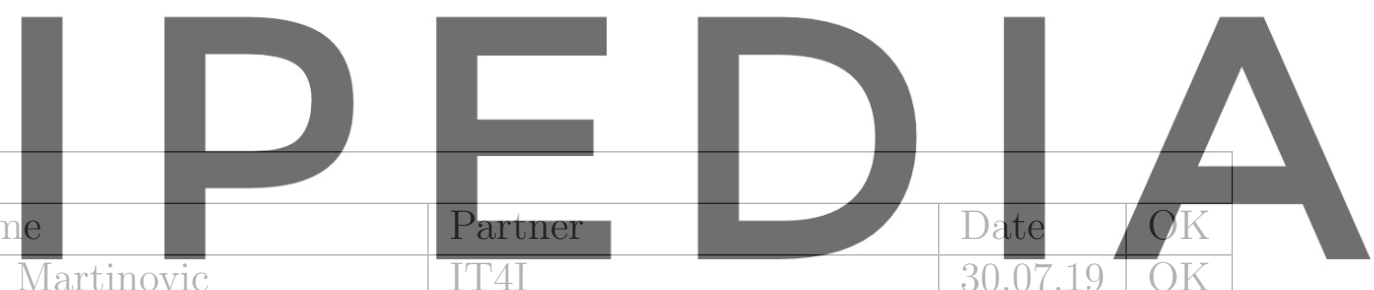

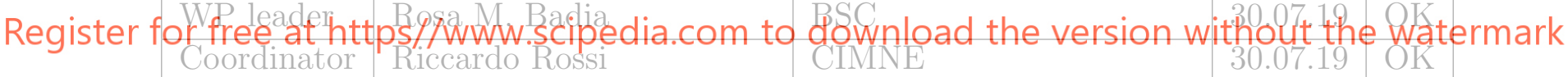




\section{Executive summary}

The main focus of this deliverable is testing and benchmarking the available infrastructure using the execution frameworks PyCOMPSs and HyperLoom. A selected benchmark employing the Multi Level Monte Carlo (MLMC) algorithm was run on two systems: TIER-0 (MareNostrum4) and TIER-1 (Salomon) supercomputers. In both systems, good performance scalability was achieved.
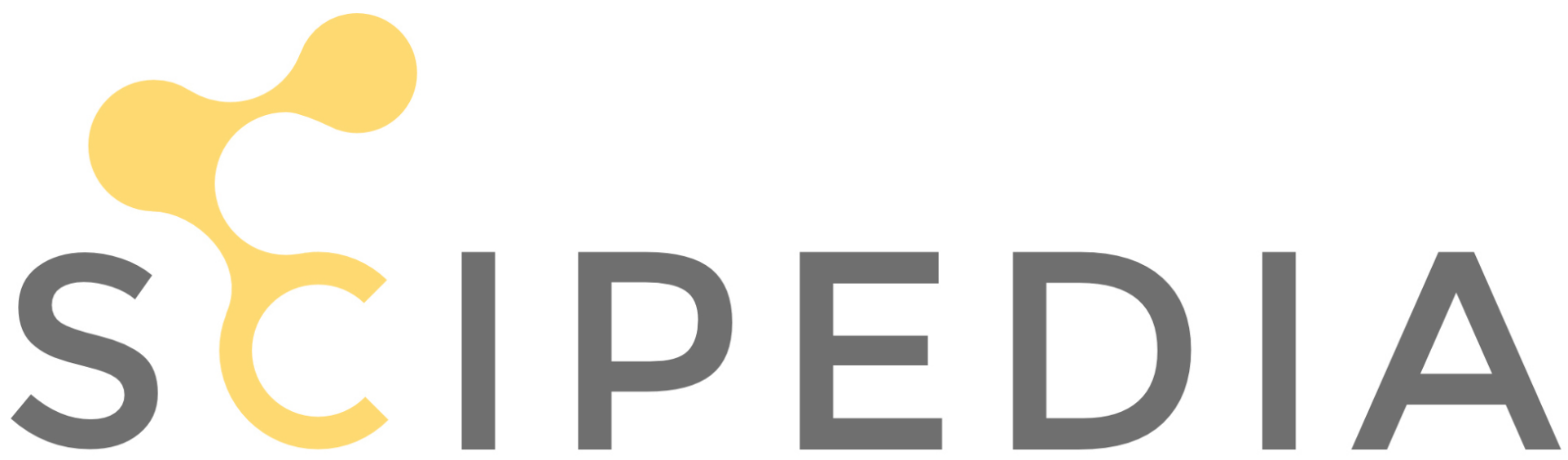

Register for free at https//www.scipedia.com to download the version without the watermark 


\section{Table of contents}

$\begin{array}{llr}1 & \text { Introduction } & 7\end{array}$

$\begin{array}{lll}2 & \text { Experiments description } & 7\end{array}$

2.1 API modification . . . . . . . . . . . . . . . . . . 8

2.1 .1 Keep flags . . . . . . . . . . . . . . . . . . . . 8

2.1 .2 Checks . . . . . . . . . . . . . . . . . 9

$2.1 .3 \operatorname{Init}() \ldots \ldots \ldots \ldots \ldots$

2.1.4 Programming style update . . . . . . . . . . . . . . 9

3 Infrastructures benchmark $\quad 10$

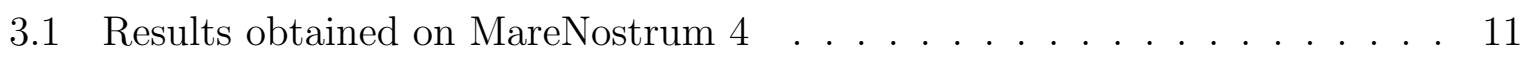

3.1 .1 PyCOMPSs ...................................... 11

3.1.2 HyperLoom

3.1.3 Conclusion .

3.2 Results obtained on Salomon .

3.2.1 PyCOMPSs

3.2.2 HyperLoom

3.2.3 Conclusion
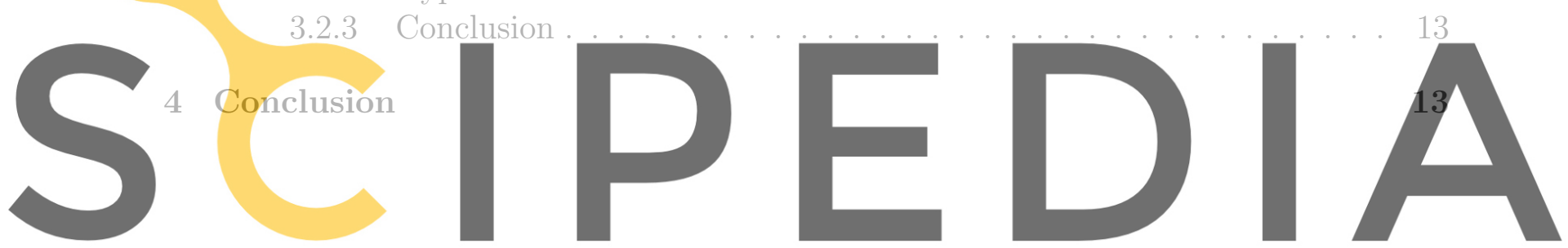

Register for free at https//www.scipedia.com to download the version without the watermark 


\section{List of Figures}

$1 \quad$ Benchmark domain $\Omega$ and boundaries. . . . . . . . . . . . . . . 7

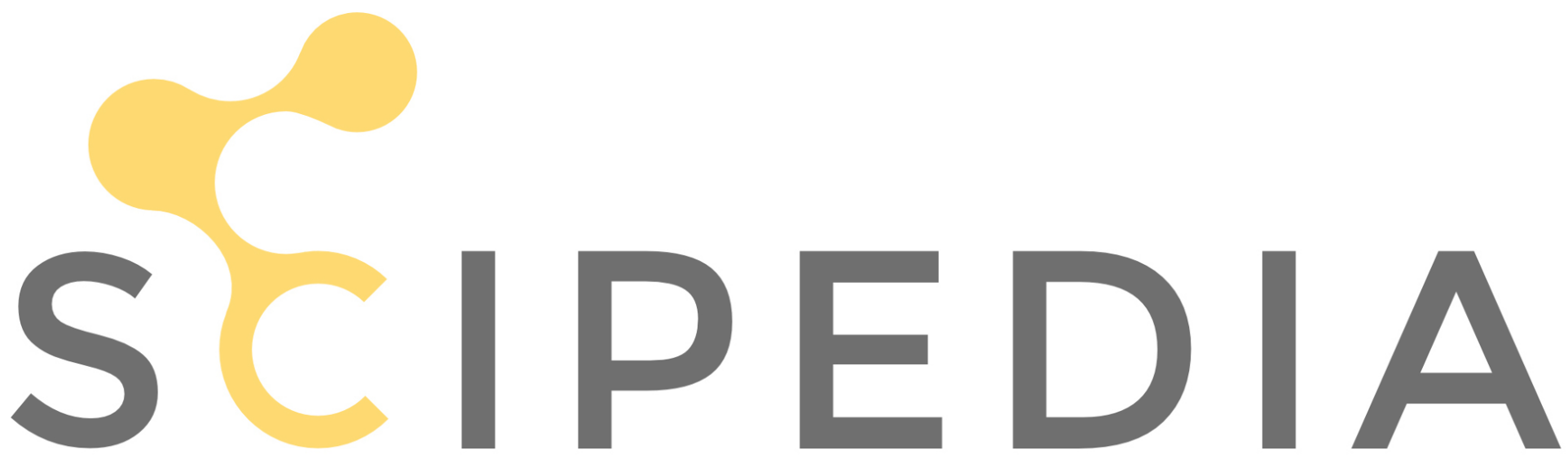

Register for free at https//www.scipedia.com to download the version without the watermark 


\section{Nomenclature / Acronym list}

\begin{tabular}{|l|l|}
\hline Acronym & Meaning \\
\hline API & Application Programming Interface \\
\hline ExaQUte & $\begin{array}{l}\text { EXAscale Quantification of Uncertainties for Technology } \\
\text { and Science Simulation } \\
\text { DArected Acyclic Graph }\end{array}$ \\
\hline FILE_IN & Path to a file passed to a function that is not modified \\
\hline FILE_INOUT & Path to a file passed to a function that is modified during the call \\
\hline FILE_OUT & Path to a file passed to a function that is created during the call \\
\hline HPC & High Performance Computing \\
\hline IN & Parameter of a function that is not modified \\
\hline INOUT & Parameter of a function that is modified during the call \\
\hline OpenMP & Open Multi Processing \\
\hline MPI & $\begin{array}{l}\text { Message Passing Interface } \\
\text { PBS }\end{array}$ \\
\hline PyCOMPSs & Python binding for COMPS Superscalar \\
\hline SLURM & Simple Linux Utility for Resource Management \\
\hline
\end{tabular}
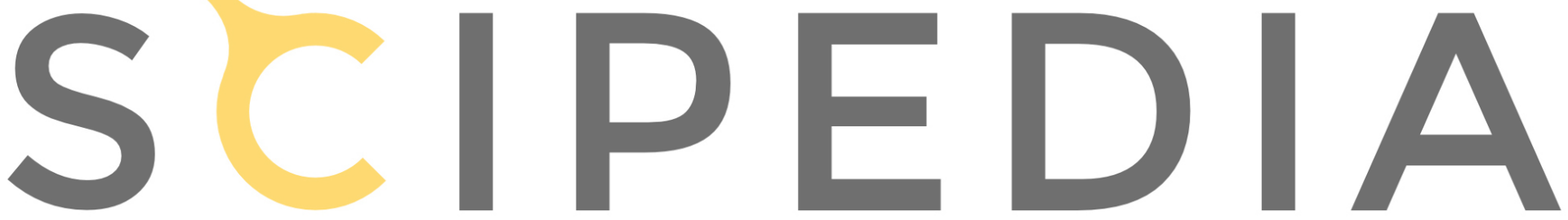

Register for free at https//www.scipedia.com to download the version without the watermark 


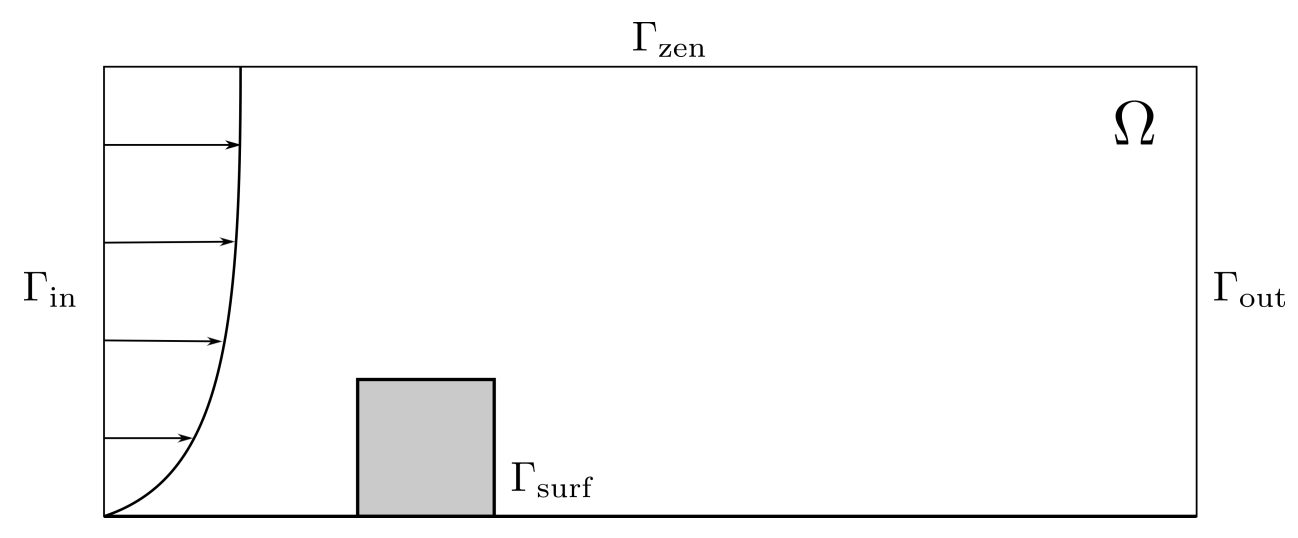

Figure 1: Benchmark domain $\Omega$ and boundaries.

\section{$1 \quad$ Introduction}

In Task 4.4, infrastructure benchmarking takes place. This deliverable summarizes the results of benchmarking on two supercomputers: MareNostrum 4 operated by BSC, and Salomon operated by IT4Innovations. The deliverable is structured as follows: In Section 2 , the benchmark run on all supercomputers is described followed by Section 3, where

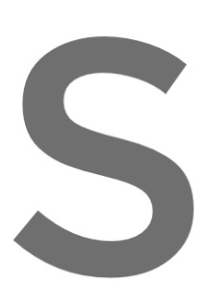
results for each super

for each supereomput

perLoom, are presenter

In Section 4, the ove
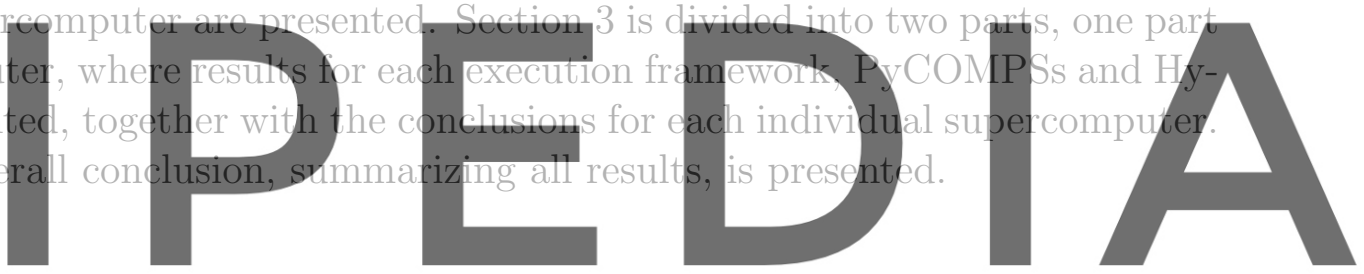

2 Experiments description

Register for free at https//www.scipedia.com to download the version without the watermark

In the ExaQUte project, the PDEs of principle concern are the incompressible Navier-

Stokes equations, given as follows:

$$
\begin{aligned}
\frac{\partial \mathbf{u}}{\partial t}+(\mathbf{u} \cdot \nabla) \mathbf{u} & =-\nabla p+\nu \nabla^{2} \mathbf{u}, \\
\nabla \cdot \mathbf{u} & =0 .
\end{aligned}
$$

In this section, we will use these equations to define a benchmark problem in order to develop the current deliverable.

In the ExaQUte project, we will be simulating wind flow past large complicated structures under calibrated physical conditions. Figure 1 is an extremely simplified 2D representation of this scenario. Here, the impeding object is simply a square, which leaves behind the computational domain $\Omega$.

On the inflow boundary, $\Gamma_{\text {in }}$, the following temporal average velocity is prescribed:

$$
\mathbf{u}\left(\mathbf{t}, \boldsymbol{\Gamma}_{\text {in }}\right) \cdot \mathbf{n}=\overline{\mathrm{u}}\left(\frac{\mathbf{z}}{\mathbf{z}_{\mathbf{0}}}\right)^{\alpha}, \quad \mathbf{u}\left(\mathbf{t}, \boldsymbol{\Gamma}_{\text {in }}\right) \cdot \mathbf{n}^{\perp}=\mathbf{0},
$$

where $\boldsymbol{n}$ is the unit normal vector field on $\partial \Omega$ and $\boldsymbol{n}^{\perp}$ is any unit length vector field orthogonal to $\boldsymbol{n}$. The novelty here is that this boundary condition is stochastic, but timeinvariant, with uncertain parameters $\overline{\mathrm{u}} \sim N(10,1.0)$ and $\alpha \sim N(0.12,0.12)$, where . The remaining boundaries, $\Gamma_{\text {surf }}, \Gamma_{\text {zen }}$, and $\Gamma_{\text {out }}$ have wall, free slip, and zero flux boundary 
conditions, respectively; i.e.,

$$
\begin{aligned}
\mathbf{u}\left(\mathbf{t}, \Gamma_{\text {surf }}\right) & =\mathbf{0}, & \boldsymbol{\sigma}\left(t, \Gamma_{\text {out }}\right) \boldsymbol{n} & =\mathbf{0}, \\
\mathbf{u}\left(t, \Gamma_{\mathrm{zen}}\right) \cdot \boldsymbol{n} & =0, & \boldsymbol{\sigma}\left(t, \Gamma_{\mathrm{zen}}\right) \boldsymbol{n} \cdot \boldsymbol{n}^{\perp} & =0,
\end{aligned}
$$

where $\boldsymbol{\sigma}=-p \mathrm{I}+\nu\left(\nabla \mathbf{u}+\nabla \mathbf{u}^{\top}\right)$ is the Cauchy stress tensor.

The above-described problem is stochastic, and the chosen algorithm to study uncertainty propagation for this deliverable is Multilevel Monte Carlo (MLMC). We refer to the work package 5 for details about the algorithm. The Quantity of Interest of study is the drag coefficient, computed on the square building.

\subsection{API modification}

For purpose of benchmarking of execution frameworks the common API defined in deliverable 4.1 is updated in the following way:

- To prevent memory leaks, intermediate temporary results were separated from objects that are requested by the user application. This has been implemented by creating a keep flag
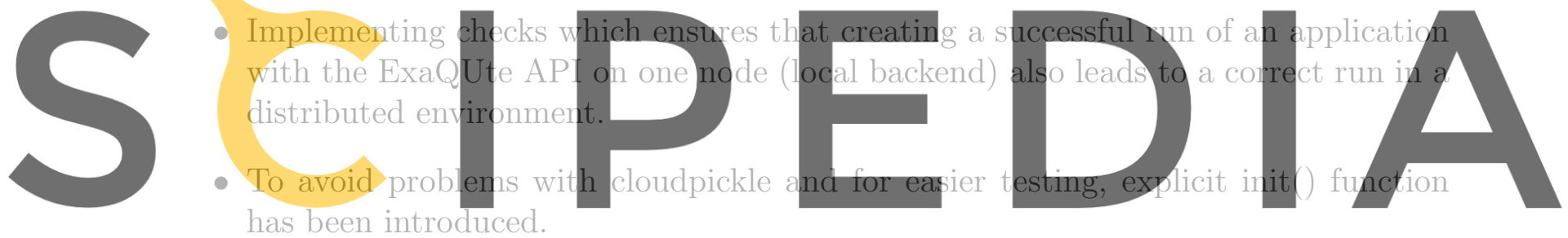

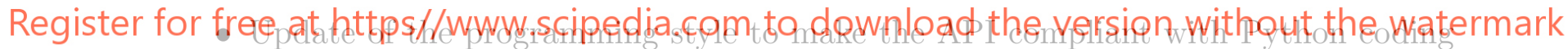
style.

\subsubsection{Keep flags}

A first improvement of the common API was the introduction of "keep" flag. If the "keep" flag is set to "true" while task is created, then it indicates that the user wants to hold the resulting value(s) of the task and is responsible for an explicit clean-up (by calling the delete_object function). Otherwise, the task result is considered temporary. In the case of chains of tasks, the results is kept until the first "synchronization point", that is, by calling "barrier" or "get_value_from_remote". At this synchronization point, the object is fully cleaned by the system when necessary and the user does not have to care about its deletion. In this case, using the object after the synchronization point returns an exception.

Example:

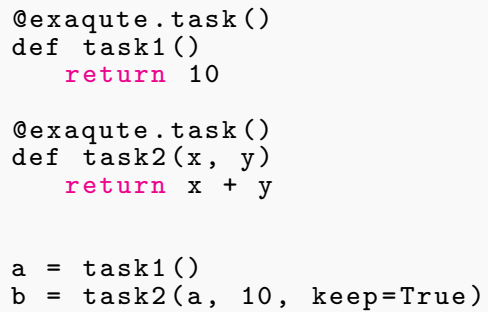




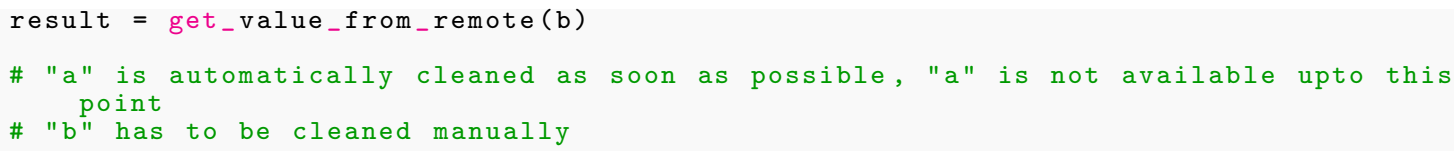

By introducing the keep flag, the invocation to the "compute" method is not required as the necessary information can be derived from the keep flags.

\subsubsection{Checks}

To ensure that creating a successful run of an application with the ExaQUte API on one node (local backend) also leads to a correct run in a distributed environment, a check procedure has been implemented.

\section{In the previous version, the "empty" implementation was used, e.g.:}

def get_value_from_remove(obj):

return obj \# just return first parameter

This solution worked and leads to functional program; however, it does not prevent an invalid usage of the API in some cases. It can also easily lead to an application that uses the API wrongly without any error. However, when a different backend is used, it may

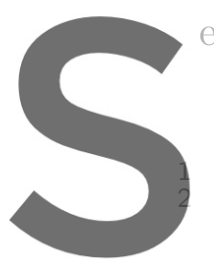
end to an error state
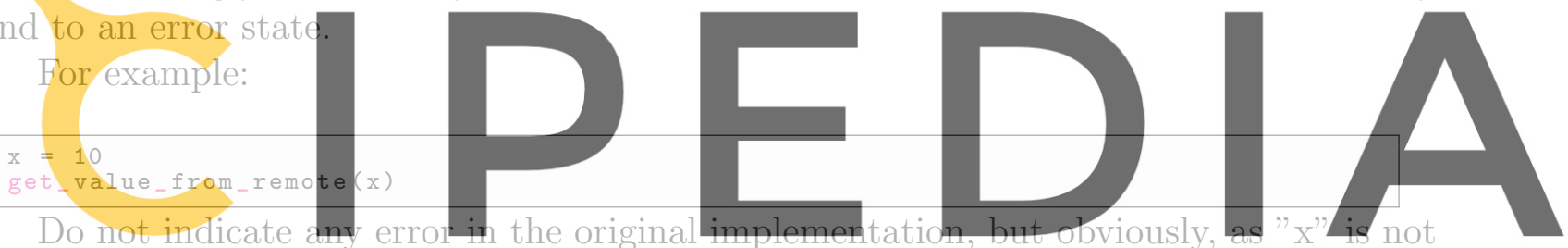

a result of task; it is an invalid usage of the API.

Register for free at https//www.scipedia.com to download the version without the watermark

\subsubsection{Init()}

The original API used an implicit initialization by the module import. This makes some simplification for users but brings some non-trivial problems.

- Modules that tracks imports usually do not assume behaviour with such a strong side effect of the import; therefore, usage of cloudpickle was broken.

- It is hard to test such libraries. It is hard to setup a test environment before import, import separated functions for unit testing without calling init, or create a fixture that initializes and deinitializes environment for each test.

So solve these problems we have introduced method init() in the ExaQUte API that contains the initialization. The user is obligated to call it before any ExaQUte function (except usage of decorators).

\subsubsection{Programming style update}

We have renamed two entities in ExaQUte API:

- ExacuteTask() to task(): It already resides in ExaQUte package, so it is not necessary to repeat this. Also, decorators are usually named by snake_case convention. 
- processing Units to processing_units: According PEP8 (standard coding style for Python), argument of a function should be named by snake case, not CamelCase convention.

\section{Infrastructures benchmark}

The following two supercomputers were used to execution the numerical experimet described in section 2 :

- The TIER-0 system MareNostrum 4 operated by BSC with 11.15 Petaflops of peak performance, which consists of 3,456 compute nodes equipped by two Intel@Xeon Platinum 8160 (24 cores at 2,1 GHz each) processors.

- The TIER-1 system Salomon operated by IT4Innovations, with 2 Petaflops of peak performance consists of 1,009 compute nodes equipped by two Intel@Xeon Haswell (12 cores at 2,5 GHz each) processors.

Those supercomputers were selected to measure the performance using the two execution frameworks (PyCOMPSs and HyperLoom) on broad range of machines because

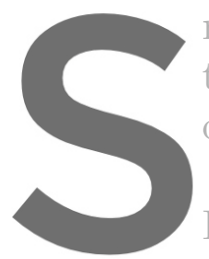
nowadays not all conlph tems and such benchn of information.

The access to the In the case of Mare Nostrum
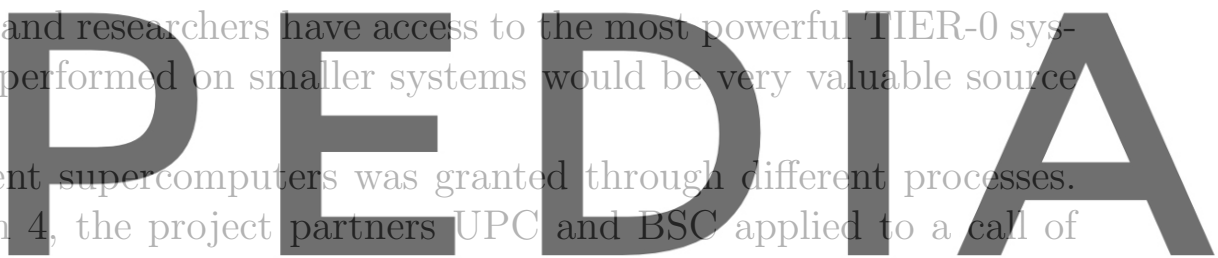

the Spanish Supercomputing Network (RES) in January 2019. The application requested

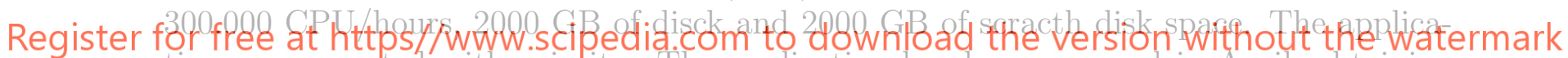
tion was accepted with priority. The application has been renewed in April, obtaining 320.000 CPU hours. In the case of Salomon, the project partners IT4I and BSC submitted proposal for multiyear project through Open Call scheme securing $1.2 \mathrm{M}$ core hours for duration of 18 months. For the next period the partners will apply for a larger allocation since the current one has been consumed very fast.

To measure the performance of the mentioned systems using the two execution frameworks, a strong scalability experiment was designed.

To equalize the differences in number of cores/node of the different systems, different number of compute nodes is defined for each system. The total number of cores in each configuration is the same. To obtain a reasonable scalability graph, four different allocations on each system has been used. The table below shows the number of compute nodes on each system.

\begin{tabular}{|l|l|}
\hline System & No Nodes \\
\hline MareNostrum & $4,8,16,32$ \\
\hline Salomon & $8,16,32,64$ \\
\hline
\end{tabular}

Table 1: Number of compute nodes used on different systems

This will result in using of $192,384,768$, and 1,536 cores in total. 
The size of the batches used in each of the Levels of the Multi Level Monte Carlo algorithm was also agreed a priori, to compare equivalent results through the different systems and different execution environments:

\begin{tabular}{|c|r|c|}
\hline Level & Batch Size & Number of OpenMP threads \\
\hline 0 & 1500 & 1 \\
\hline 1 & 125 & 2 \\
\hline 2 & 10 & $\begin{array}{c}\text { 4 or optionally, the number of cores } \\
\text { available in the node }\end{array}$ \\
\hline
\end{tabular}

Table 2: Batch size for different levels of MLMC

\subsection{Results obtained on MareNostrum 4}

Since the MLMC algorithm is asynchronous and the possibility of exploiting this fact is very important, we have chosen to run 9 full iterations with 4 active batches at each time. We also decided to run second benchmark with more batches in each level of MLMC on MareNostrum 4 (see table 3).

\begin{tabular}{|c|r|c|}
\hline Level & Batch Size & Number of OpenMP threads \\
\hline 0 & 2000 & 1 \\
\hline 1 & 150 & 2 \\
\hline 2 & 10 & $\begin{array}{c}\text { 4 or optionally, the number of cores } \\
\text { available in the node }\end{array}$ \\
\hline
\end{tabular}

Table 3: Batch size for different levels of MLMC on MareNostrum 4

\subsubsection{PyCOMPSs}

Table 4 shows the results with the batch size $[1500,125,10]$ described at the beginning of this chapter. Table 5 shows the results for second benchmark with batch size [2000, 150, 10].

\subsubsection{HyperLoom}

To be able to compare the results obtained on Salomon, the same version on HyperLoom was installed on MareNostrum as well. During the testing we encountered problem by

\begin{tabular}{|c|r||r|c||c|c|}
\hline No of nodes & No of cores & Execution time (s) & Speedup & Ideal Speeedup & $\%$ \\
\hline \hline 4 & 192 & 36947 & 1.00 & 1 & 1.00 \\
\hline 8 & 384 & 19325 & 1.91 & 2 & 0.96 \\
\hline 16 & 768 & 10721 & 3.45 & 4 & 0.86 \\
\hline 32 & 1536 & 5822 & 6.35 & 8 & 0.79 \\
\hline
\end{tabular}

Table 4: PyCOMPSs results with batch size of $[1500,125,10]$ in Mare Nostrum 


\begin{tabular}{|c|r||r|c||c|c|}
\hline No of nodes & No of cores & Execution time (s) & Speedup & Ideal Speedup & $\%$ \\
\hline \hline 4 & 192 & 48143 & 1.00 & 1 & 1.00 \\
\hline 8 & 384 & 24790 & 1.94 & 2 & 0.97 \\
\hline 16 & 768 & 13019 & 3.70 & 4 & 0.93 \\
\hline 32 & 1536 & 7273 & 6.62 & 8 & 0.83 \\
\hline 64 & 3072 & 4089 & 11.78 & 16 & 0.74 \\
\hline 128 & 6144 & 2742 & 17.56 & 32 & 0.55 \\
\hline
\end{tabular}

Table 5: PyCOMPSs results with batch size of $[2000,150,10]$ in MareNostrum

executing the Problem_zero use-case. We investigated this problem and we were able to track down the problem to the point of transferring the computation to a worker. In HyperLoom, the commonly used module "cloudpickle" is used to serialize computation. The problem when running the Problem_zero use-case is, that when the computation is serialized and later on de-serialized a function that is using Kratos, the Python interpreter get stucked. Unfortunately we were not able to construct a smaller example to debug it further. We tested Kratos and HyperLoom independently and both were working fine. Also, the de-serialization of any other object different to a Kratos involved function was working and the de-serialization of such object outside of Loom was working as well. It should be noted that we have been running the same configuration on Salomon and we never encountered such a problem. Moreover, the de-serialization in "cloudpickle" is quite lightweight, so we could rule it out as a root cause of the problem.

We were unable to investigate it further because unfortunately, the debugging tool Valgrind is not working on MareNostrum, so we were not able to use Helgrind or DRD to use these tools for detecting lock violations. With a plain GDB tool, we are able to see that is hanging somewhere in the Python interpreter, but without any futher details. We have asked MareNostrum support to install Python debuginfo package that should provide us with insight on what is happening in Python. MareNostrum support installed Python debuginfo package in version 3.6.6. Since Kratos was instaled on MareNostrum using version 3.6.1 of Python, it has to be re-builded using same version of the Python i.e. 3.6.6. Unfortunately new problems arises during installation of Kratos which are being currrently being solved by MareNostrum support.

\subsubsection{Conclusion}

Results obtained on MareNostrum 4 by PyCOMPSs shows very good scalability of the example with batch sizes $[1500,125,10]$.

With the second batch size $[2000,150,10]$ we observe that with 128 nodes the efficiency decreases in a larger proportion than before with smaller node-counts. It would be interesting to test a highest batch size since we are at the limit of filling the computer with enough computation.

\subsection{Results obtained on Salomon}

Since Salomon is much smaller machine than MareNostrum 4 we run a smaller case with a batch size of $[1500,125,10]$ and with an alternative number of iterations. With PyCOMPSs we setthe number of iterations to 9 and experiment executed by HyperLoom 


\begin{tabular}{|c|r||r|c||c|c|}
\hline No of nodes & No of cores & Execution time (s) & Speedup & Ideal Speedup & $\%$ \\
\hline \hline 4 & 96 & 66705 & 1.00 & 1 & 1.00 \\
\hline 8 & 192 & 32780 & 2.03 & 2 & 1.01 \\
\hline 16 & 384 & 18289 & 3.65 & 4 & 0.91 \\
\hline 32 & 768 & 10164 & 6.56 & 8 & 0.82 \\
\hline 64 & 1536 & 7068 & 9.44 & 16 & 0.40 \\
\hline
\end{tabular}

Table 6: PyCOMPSs results with batch size of $[1500,125,10]$ in Salomon (9 iterations)

\begin{tabular}{|c|r||r|c||c|c|}
\hline No of nodes & No of cores & Execution time (s) & Speedup & Ideal Speeedup & $\%$ \\
\hline \hline 4 & 96 & 25171 & 1.00 & 1 & 1.00 \\
\hline 8 & 192 & 12376 & 2.03 & 2 & 1.02 \\
\hline 16 & 384 & 6014 & 4.18 & 4 & 1.05 \\
\hline 32 & 768 & 4673 & 5.38 & 8 & 0.67 \\
\hline 64 & 1536 & 3859 & 6.52 & 16 & 0.41 \\
\hline
\end{tabular}

Table 7: HyperLoom results with batch size of $[1500,125,10]$ in Salomon (2 iterations)

used 2 iterations. In both cases we keep number of active batches the same as we run on MareNostrum 4 (4 batches).

\subsubsection{PyCOMPSs}

Table 6 shows the results with the batch size $[1500,125,10]$ and 9 iterations performed by PyCOMPs on Salomon supercomputer.

\subsubsection{HyperLoom}

Times obtained by running experiment with batch size $[1500,125,10]$ and 2 iterations are reported in Table 7 .

\subsubsection{Conclusion}

Both cases run on Salomon supercomputer shows very good scalability, close to ideal one, up to the 16 compute nodes i.e. 384 cores. After this, the scalability drops significantly. From presented results could be concluded that even for TIER-1 system much bigger case could be designed and run.

\section{Conclusion}

Results obtained on both systems, MareNostrum 4 (TIER-0) and Salomon (TIER-1), exhibit very good scalability up to a certain. However, speedup decrease could be explained by the size of the benchmark which do not have enough computation workload for such large machines.

Results also show that there is no large difference between TIER-0 and TIER-1 systems when it comes the the scalability of presented benchmarks. Both systems shows good 
scalability up to the 768. In the future benchmarking will continue with bigger batches and bigger examples which will on finest level of MLMC run on multiple compute nodes. 